\title{
Decay spectroscopy of exotic nuclei at RIBF
}

\author{
H. Watanabe ${ }^{1,2}$ \\ ${ }^{1}$ IRCNPC, School of Physics and Nuclear Energy Engineering, Beihang University, China \\ ${ }^{2}$ RIKEN Nishina Center
}

\begin{abstract}
Neutron-rich nuclei around the doubly magic nucleus ${ }^{132} \mathrm{Sn}$ and the double midshell nucleus ${ }^{170}$ Dy have been investigated by means of decay spectroscopy techniques with the EURICA setup at the RIBF facility at RIKEN. The nuclei of interest were produced by in-flight fission of a high-intensity ${ }^{238} \mathrm{U}$ beam at $345 \mathrm{MeV} / \mathrm{u}$. In this contribution, some selected topics are reported.
\end{abstract}

\section{Introduction}

Exotic nuclei far from the valley of $\beta$ stability can serve as a good probe for studying new phenomena, such as neutron skin/halo structures and appearance/disappearance of magic numbers, which emerge with largely asymmetric values of the $N / Z$ ratio. The advent of the third-generation in-flight fragment separator facility, the RI-Beam Factory (RIBF) at RIKEN [1], enables systematic investigations of such novel nuclear properties and allows for extension of our knowledge of nuclear structures towards the neutron and proton drip lines. In that occasion, $\gamma$-ray spectroscopy following the $\beta$ decay is an effective tool for probing low-lying yrast and non-yrast states, from which key information on nuclear structure, such as shape transitions/coexistence and single-particle orbits, can be obtained. For the study of rare isotopes, especially when the nucleus of interest lies at the boundaries of availability for spectroscopic studies, isomeric decays are likely to be a more useful means than $\beta$ decays to populate excited levels. The combined $\beta-\gamma$ and isomeric-decay measurements using state-of-the-art detection systems at RIBF, which has the capability of providing the world's strongest RI beams, are at the forefront of exploration of exotic nuclei far from stability.

The RIBF was commissioned in 2007, with the adoption of a sequential acceleration system consisting of a linac injector (RILAC) and four ring cyclotrons (RRCfRC-IRC-SRC), which can provide heavy-ion beams at $345 \mathrm{MeV} / \mathrm{u}$. The primary-beam intensity increases year by year owing to the steady improvement of the accelerators and the related devices. For instance, the intensity of a ${ }^{238} \mathrm{U}$ beam was lower than $0.1 \mathrm{pnA}$ when the facility was commissioned, but now it can be delivered with a stable intensity higher than 20 pnA. The first decay spectroscopy experiment at RIBF was carried out in 2009 with four Compton-suppressed Clover-type Ge detectors arranged around an active stopper system consisting of nine double-sided silicon-strip detectors (DSSSD) stacked compactly. A major aim of this work was the study of neutron-rich nuclei around $Z=40$ and $A=110$, where the shape transitions from prolate, via $\gamma$-soft, to oblate deformations are predicted to occur with increasing the number of neutrons. New results obtained include the observation of shape evolution in ${ }^{106,108} \mathrm{Zr}$ [2], a possible oblateshape isomer in ${ }^{109} \mathrm{Nb}$ [3], and a large-amplitude $\gamma$-soft dynamics in ${ }^{110} \mathrm{Mo}$ [4]. These findings revealed the shapetransitional phenomena in this neutron-rich region for the first time.

Research opportunities for decay spectroscopy at RIBF can be expanded in the EURICA (EUROBALLRIKEN Cluster Array) project [5]. The main body of EURICA consists of 12 Cluster-type HPGe detectors, surrounding a highly segmented silicon stopper system named WAS3ABi [5]. In addition to the normal EURICA setup, $18 \mathrm{LaBr}_{3}(\mathrm{Ce})$ detectors and plastic scintillators are installed for the fast-timing measurement of $\gamma$ and $\beta$ rays, respectively. The aim of EURICA is to pin down currently controversial subjects in nuclear physics and nuclear astrophysics, such as the evolution of shell structures that can lead to the appearance or disappearance of the spherical magic numbers, the effect of weak binding and pairing in largely diffused neutron densities, search for stable oblate, triaxial, and higher-order deformations at low excitation energy, and the decay properties of neutron-rich isotopes relevant to the $r$-process nucleosynthesis. A wide range of unstable nuclei on the Segre chart are within the scope of EURICA. In this report, some selected results of the EURICA experimental campaigns will be introduced, highlighting $\gamma$-ray spectroscopy of exotic nuclei in the vicinity of doubly magic ${ }^{132} \mathrm{Sn}$ and doubly mid-shell ${ }^{170} \mathrm{Dy}$.

\section{Selected topics from EURICA}

\subsection{Isomeric states in ${ }^{126} \mathrm{Pd}_{80}$ and ${ }^{128} \mathrm{Pd}_{82}$}

Figure 1(a) shows a $\gamma$-ray energy spectrum measured in delayed coincidence with ${ }^{128} \mathrm{Pd}$ ions. Four $\gamma$ rays at ener- 
gies of $75,260,504$, and $1311 \mathrm{keV}$ have been unambiguously observed. These $\gamma$ rays are found to be in mutual coincidence and exhibit consistent time behavior. Therefore, we conclude that they proceed through a single cascade originating from one isomeric state. A least-squares fit of the summed gated time spectra of the isomeric-decay transitions yields $T_{1 / 2}=5.8(8) \mu \mathrm{s}$. The total internal conversion coefficient $\left(\alpha_{T}\right)$ for the $75-\mathrm{keV}$ transition derived from a comparison with the $1311-\mathrm{keV} \gamma$-ray intensity is $2.6(17)$, which is consistent with the theoretical value of $\alpha_{T}=3.88$ for an E2 multipolarity. Based on these arguments, the level scheme of ${ }^{128} \mathrm{Pd}$ is proposed as displayed in Fig. 2, where the spin and parity of the $5.8-\mu$ s isomeric state at $2151 \mathrm{keV}$ is assigned as $J^{\pi}=8^{+}$. A transition strength of $B\left(E 2 ; 8^{+} \rightarrow 6^{+}\right)=0.22(3)$ W.u. can be obtained from the measured half-life of the $2151-\mathrm{keV}$ isomeric state.

Assuming that the excited states in ${ }^{128} \mathrm{Pd}$ are the same multiplet members (with seniority $v=2$, as will be discussed later), the half-life of the $J^{\pi}=6^{+}$state is estimated to be $22(3) \mathrm{ns}$ from that of the $8^{+}$isomer. Since this value is significantly shorter than the flight time of fragments from the production target to the implantation position ( $650 \mathrm{~ns}$ ), there would be little ${ }^{128} \mathrm{Pd}$ ions remaining at this state during the flight. Hence, the observed intensities of the four cascade $\gamma$ rays should depend only on the population of the $8^{+}$isomeric state.

The excitation energies of the $J^{\pi}=2^{+}-8^{+}$states in ${ }^{128} \mathrm{Pd}$ are comparable to those in ${ }^{130} \mathrm{Cd}$ [6]. The constancy of level energies is characteristic of the seniority scheme, where seniority $v$ counts the number of nucleons that are not in pairs coupled to spin zero. In case of a $n$-particle (or $n$-hole) system in a single- $j$ shell, the level energies with identical $J^{\pi}$ and $v$ are independent of $n$. Thus, the excited states in ${ }^{128} \mathrm{Pd}$ can be interpreted in terms of the $v=2$ configuration of the $\pi g_{9 / 2}$ subshell, which locates just below the $Z=50$ shell gap.

A direct implication of the $N=82$ shell closure can be corroborated from the behavior of the $E 2$ transition strength for the $8^{+} \rightarrow 6^{+}$isomer decay in ${ }^{128} \mathrm{Pd}$. The $E 2$ matrix elements between $v=2$ states are close to zero near the middle of the valence shell in semi-magic nuclei, giving rise to seniority isomerism. For the $N=82$ isotones, it turns out that the $B\left(E 2 ; 8^{+} \rightarrow 6^{+}\right)$value is much smaller in ${ }^{128} \mathrm{Pd}$ than in ${ }^{130} \mathrm{Cd}$, as expected in the exact seniority classification, namely, $B(E 2 ; v=2, n=4)=\frac{1}{9} B(E 2 ; v=$ $2, n=2)$. This observation indicates that both the $J^{\pi}=8^{+}$ and $6^{+}$states in the $N=82$ isotones have good seniority $v=2$ in the well isolated $\pi g_{9 / 2}$ subshell, in consequence of the robust shell closure.

The level scheme of ${ }^{126} \mathrm{Pd}$ constructed in the current work is also shown in Fig. 2. The 693-keV $\gamma$ ray, which is the most intense peak of those observed in delayed coincidence with ${ }^{126} \mathrm{Pd}$ ions [Fig. 1(b)], is assigned as the $2^{+} \rightarrow 0^{+}$transition. The coincidence spectrum with a gate on the 542-keV $\gamma$ ray clearly exhibited $\gamma$ rays at energies of 693,788 , and $86 \mathrm{keV}$, but not at $1330 \mathrm{keV}$, indicating that two parallel cascades stem from a common level at 2023 $\mathrm{keV}$. The $86-\mathrm{keV}$ transition is placed just above the 2023 -
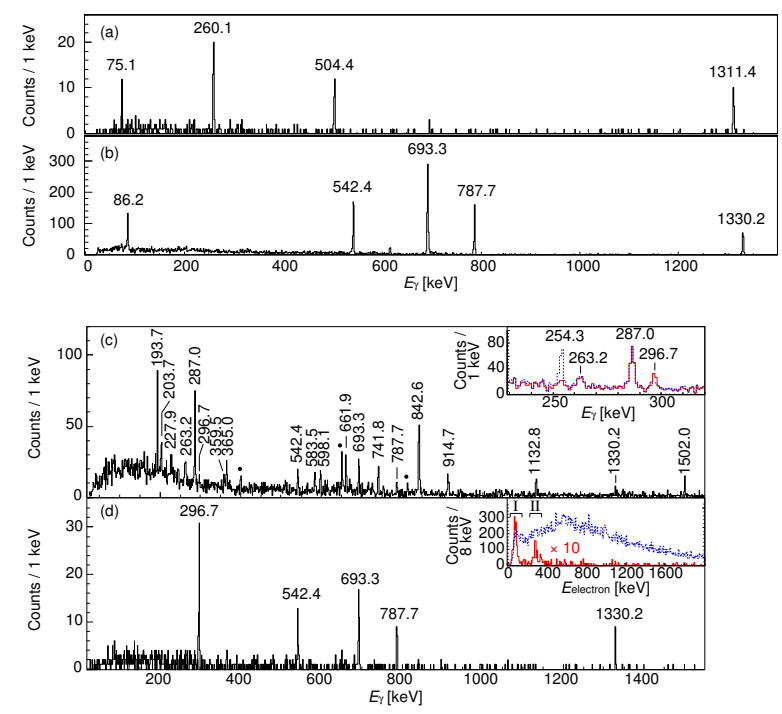

Figure 1. Gamma-ray spectra measured (a) in coincidence with ${ }^{128} \mathrm{Pd}$ ions within a time range of $0.15-25 \mu \mathrm{s}$, (b) in coincidence with ${ }^{126} \mathrm{Pd}$ ions within $0.15-5 \mu \mathrm{s}$, and (c) within $50 \mathrm{~ms}$ after the ${ }^{126} \mathrm{Pd}$ implantation with a gate on an electron- $\gamma$ time difference of $-0.5 \leq \Delta t_{e \gamma} \leq 0.5 \mu \mathrm{s}$. Contaminants from the granddaughter ${ }^{126} \mathrm{Cd}$ are marked with filled circles. The inset magnifies the energy region from 230 to $310 \mathrm{keV}$ measured with $-4.0 \leq \Delta t_{e \gamma} \leq$ $0.5 \mu \mathrm{s}$ (red) and $-0.5 \leq \Delta t_{e \gamma} \leq 50 \mu$ s (blue). (d): $\gamma$ rays measured with $-4.0 \leq \Delta t_{e \gamma} \leq 0.5 \mu \mathrm{s}$, and additionally, with a gate on the electron peak marked with "I" in the inset, where the spectrum depicted with the red line (multiplied by a factor of ten) is obtained with a sum of gates on the $\gamma$ rays below the $\left(5^{-}\right)$isomer in ${ }^{126} \mathrm{Pd}$, while the blue line represents an electron spectrum without $\gamma$-ray gates.

$\mathrm{keV}$ level, because this $\gamma$ ray was observed to precede all the four transitions.

A half-life of $0.44(3) \mu$ s is derived from the time distribution of the $86-\mathrm{keV} \gamma$ ray relative to the beam implantation, while the time difference between the $86-\mathrm{keV} \gamma$ ray and the subsequent transitions provides $T_{1 / 2}=0.33(4)$ $\mu \mathrm{s}$. Thus, it is found that two isomeric states lie at 2023 and $2110 \mathrm{keV}$. The assignment of spins and parities for the two isomeric states is based on the $\gamma$-ray feeding pattern and transition probabilities. Given the $J^{\pi}=5^{-}$ and $7^{-}$assignments for the 2023- and 2110-keV levels, respectively, the strengths of the isomeric decay transitions are $B\left(E 1 ; 5^{-} \rightarrow 4^{+}\right)=2.9(4) \times 10^{-9}$ W.u. $(542$ $\mathrm{keV}), B\left(E 3 ; 5^{-} \rightarrow 2^{+}\right)=0.24(3)$ W.u. $(1330 \mathrm{keV})$, and $B\left(E 2 ; 7^{-} \rightarrow 5^{-}\right)=2.13(14)$ W.u. $(86 \mathrm{keV})$. These strengths are comparable with the values of the corresponding transitions in the $N=80$ isotone ${ }^{128} \mathrm{Cd}$ [7].

The $\gamma$ rays below these isomers, except for the $86-\mathrm{keV}$ line, have been also observed in coincidence with electrons that were associated with the prior implantation of ${ }^{126} \mathrm{Pd}$, as demonstrated in Fig. 1(c). This observation implies the existence of a long-lived, higher-spin isomer which decays via the cascades that include electromagnetic transitions with relatively large total conversion coefficients. With gates on these $\gamma$ rays, a prominent peak can be found in an electron spectrum [marked with "I" in the inset of 

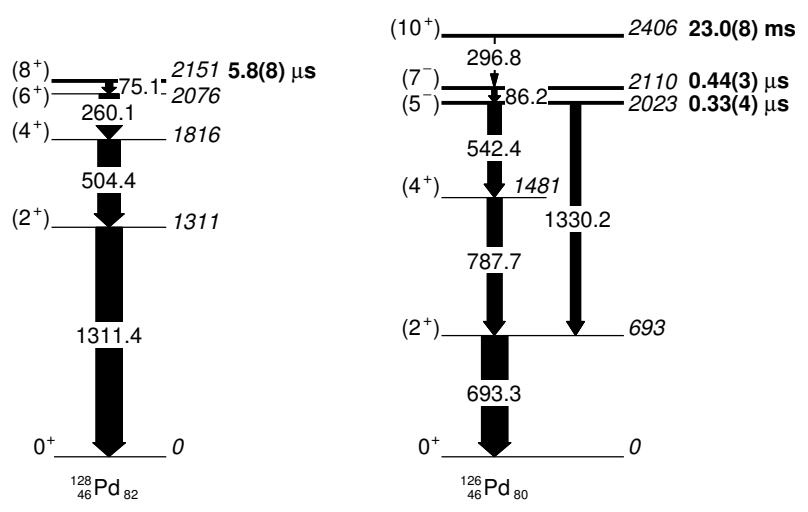

Figure 2. Partial level schemes of ${ }^{128} \mathrm{Pd}$ (left) and ${ }^{126} \mathrm{Pd}$ (right) established in the present work.

Fig. 1(d)]; this corresponds to the conversion electrons for the 86-keV, E2 transition ( $\left.\alpha_{T}=2.374\right)$. In Fig. 1(d), a $\gamma$ ray at $297 \mathrm{keV}$ is clearly visible in addition to the $\gamma$ rays below the $\left(5^{-}\right)$isomer by gating on the $86-\mathrm{keV}$ IC peak. The appearance of the $297-\mathrm{keV}$ peak is emphasized by taking a $\gamma$-ray time condition earlier than electron events, as is evident from the inset of Fig. 1(c), suggesting that this new $\gamma$ ray precedes the highly converted $86-\mathrm{keV}$ transition. Thus, the long-lived isomer can be identified at an excitation energy of $2406 \mathrm{keV}$. A peak marked with "II" in the inset of Fig. 1(d) is expected to arise from the conversion electrons for the 297-keV transition, being most likely of an $E 3$ character $\left(\alpha_{T}=0.1197\right)$. In addition to the internaldecay branch, $\beta$ decay from the long-lived isomer was observed to populate excited states at high spins in ${ }^{126} \mathrm{Ag}$. The isomeric half-life was determined to be $23.0(8) \mathrm{ms}$ by taking a weighted average of those derived from the time distributions of the internal- and external-decay branches.

The main surprise in the present work is the small energy difference between the $\left(10^{+}\right)$and $\left(7^{-}\right)$isomers, $\Delta E_{7^{-}}^{10^{+}}$, in ${ }^{126} \mathrm{Pd}$, compared to the analogous levels in the heavier $N=80$ isotones, as demonstrated in Fig. 3 . Since these two levels consist predominantly of maximally aligned two neutron-hole configurations, $\left(v 1 h_{11 / 2}^{-2}\right)_{10^{+}}$and $\left(v 1 h_{11 / 2}^{-1} 2 d_{3 / 2}^{-1}\right)_{7^{-}}$, their level energies depend on the singleparticle energies (SPEs) of the $v 1 h_{11 / 2}$ and $v 2 d_{3 / 2}$ neutron orbits, as well as the strength of interactions between them. It can be seen in Fig. 3 that the energy of the $11 / 2^{-}$level relative to the $3 / 2^{+}$ground state in the neighboring $N=81$ isotones decreases when approaching $Z=50$. Note that these states are of one neutron-hole nature, and that the smooth reduction in energy can be essentially interpreted in terms of a short-range proton-neutron interaction as follows: The $\pi 1 g_{7 / 2}$ proton orbit lies just above the $Z=50$ shell closure. The monopole interaction between $\pi 1 g_{7 / 2}$ and $v 1 h_{11 / 2}$, a spin-flip pair with $\Delta \ell=1$ and $\Delta n=0$, is stronger than the $\pi 1 g_{7 / 2}-v 2 d_{3 / 2}$ pair, due to the larger overlap between the radial wave functions of the two orbits. Therefore, when emptying the $\pi 1 g_{7 / 2}$ subshell, the $v 1 h_{11 / 2}$ orbit is relatively less bound than the $v 2 d_{3 / 2}$ one. The moderation in the slope at higher $Z$ may be ascribed

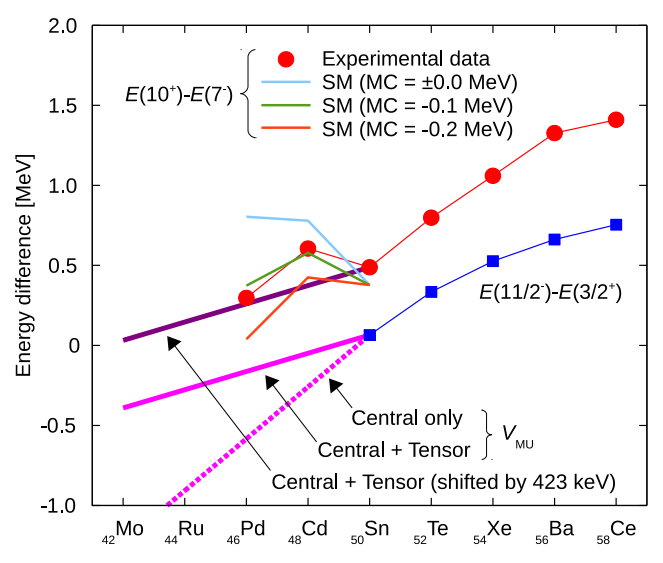

Figure 3. (color online). Energy differences between the $10^{+}$ and $7^{-}$states, $\Delta E_{7^{-}}^{10^{+}}$, in $N=80$ (filled circles) and between the $11 / 2^{-}$and $3 / 2^{+}$states in $N=81$ (filled squares). For $Z \leq 50$, the differences in energy between the $1 h_{11 / 2}$ and $2 d_{3 / 2}$ neutron orbits calculated by $V_{\mathrm{MU}}$ interaction [8] and $\Delta E_{7^{-}}^{10^{+}}$by shell-model (SM) calculations with monopole corrections (MC) are also depicted.

to the partial occupation of the $\pi 2 d_{5 / 2}$ orbit, which exerts a weaker (stronger) monopole interaction on the $v 1 h_{11 / 2}$ $\left(v 2 d_{3 / 2}\right)$ neutrons than $\pi 1 g_{7 / 2}$. As is evident from the comparison in Fig. 3, a similar trend is observed for $\Delta E_{7^{-}}^{10^{+}}$. This finding supports that these isomers can serve as sensitive probes for the evolution of the constituent neutron shell orbits.

Below $Z=50$, protons in the $\pi 1 g_{9 / 2}$ subshell play a major role in changing the neutron SPEs, because this orbit is near the Fermi surface. The neutron SPEs are estimated by the monopole-based universal interaction, $V_{\mathrm{MU}}$, which consists of the Gaussian central force and the tensor force based on the $\pi+\rho$ meson exchanges, using the parameters fixed in Ref. [8]. The $V_{\mathrm{MU}}$ interaction has been applied to other regions of exotic nuclei and successfully reproduced various types of the shell evolution [8]. Figure 3 shows the evolutions of the relative $11 / 2^{-}$energy starting from the experimental value in ${ }^{131} \mathrm{Sn}$, predicted by the $V_{\mathrm{MU}}$ calculation with $A=130$. In the calculation, the $\pi 1 g_{9 / 2}$ subshell is considered to be well isolated between $Z=50$ and 40. In Fig. 3, only with the central force the $11 / 2^{-}$energy rapidly decreases to negative values as the proton number decreases, indicating that the $v 1 h_{11 / 2}$ orbit moves away from the $v 2 d_{3 / 2}$ one towards the $N=82$ gap. If there is the tensor force effect, however, the slope becomes less steep. The observed reduction in $\Delta E_{7^{-}}^{10^{+}}$from ${ }^{130} \mathrm{Sn}$ to ${ }^{126} \mathrm{Pd}$ is consistent with the expectation of $V_{\mathrm{MU}}$ including both the central and tensor forces, given the same trend as the $11 / 2^{-}$relative energies. Based on these findings, it can be concluded that the tensor force slows down the upward drift of the $v 1 h_{11 / 2}$ SPE when protons are removed from the $\pi 1 g_{9 / 2}$ orbit below ${ }^{132} \mathrm{Sn}$. 


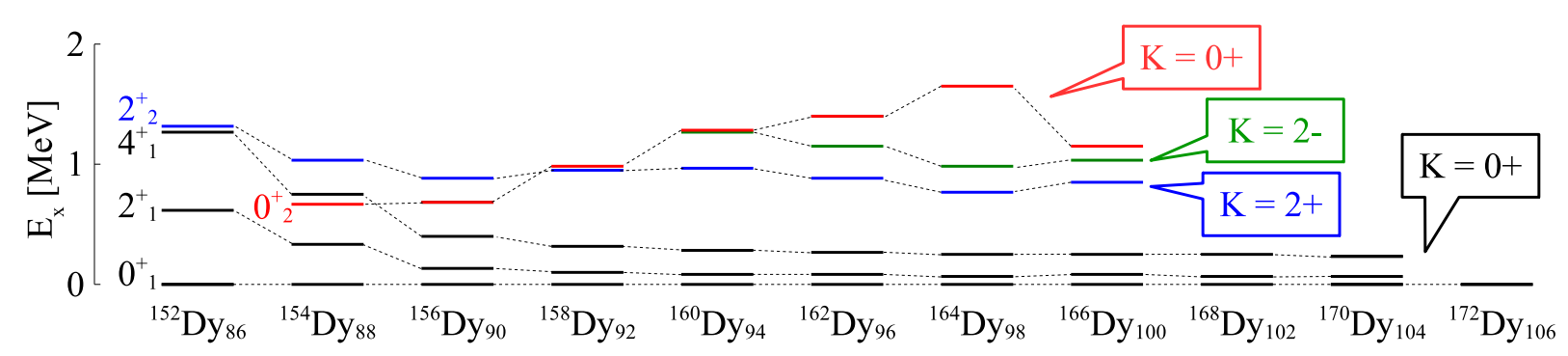

Figure 4. Systematic of the low-lying levels in even-even Dy isotopes.

\subsection{Decay spectroscopy of neutron-rich rare-earth isotopes}

It is well known that rare-earth isotopes beyond $N=90$ have well-deformed prolate shapes. According to the fundamental phenomenon of the nuclear collectivity being enhanced as the number of valence nucleons increases, it is conjectured that the maximum ground-state deformation occurs in dysprosium-170 ( $\left.{ }^{170} \mathrm{Dy}: Z=66, N=104\right)$, which lies at the middle of the major shells for both proton and neutron between the well-established doubly closedshell isotopes ${ }^{132} \mathrm{Sn}(Z=50, N=82)$ and ${ }^{208} \mathrm{~Pb}(Z=82$, $N=126)$. In the actual nuclei, however, the stability of shape should be influenced by the presence of sub-shell closures, residual interactions, and possibly, the neutron excess. Therefore, well-deformed nuclei in this neutronrich region can serve as a benchmark for testing nuclear mean-field model calculations.

Concerning astrophysical interest, it has been suggested that the decay properties of deformed nuclei in this neutron-rich region, such as neutron emission probabilities and $\beta$-decay rates, play a significant role in forming a pygmy peak around $A=160$ in the $r$-process solar abundance distribution [9]. This is the so-called the rare-earth element (REE) peak, which appears between the prominent $r$-process peaks at $A \approx 130$ and 195. While it is understood that the main $r$-process peaks are due predominantly to the "waiting point" nuclei arising from the competition between slow neutron-capture rates and long $\beta$ decay lifetimes at the neutron closed shells, the REE peak appears far away both from the proton and neutron shell closures, suggesting the presence of a different mechanism for its formation. In this context, the nuclear deformation in this neutron-rich region is considered to significantly affect the REE peak formation. However, it is still unclear how the nuclear shape evolves and where the deformation maximum takes place in the double midshell region because spectroscopic information on the excited states remains scarce due to the difficulties in access to this neutron-rich region. Theoretical models that are often used as input for $r$-process calculations can not reproduce the experimentally measured deformations very well. Thus, the REE peak can serve as a unique probe of the $r$ process nucleosynthesis, despite its less abundance than the other major peaks at $A \approx 130$ and 195 , and better knowledge on the evolution of collectivity in this region is required.
The major aim of the present work is to scrutinize the structural evolution of Dy isotopes in the double midshell region by means of $\gamma$-ray spectroscopy following isomer and $\beta$ decays, with particular attention to the physics cases and observables characteristic of deformed nuclei as outlined below:

\section{Low-lying levels in even-even isotopes}

Spectroscopic study of low-lying levels in even-even nuclei provides significant information on nuclear shapes and the resulting collective motions. According to the global systematics, it is empirically known that the deformation parameter $\beta$ is inversely proportional to the square root of the first $2^{+}$level energy $E\left(2_{1}^{+}\right)$[10], while the $R_{4 / 2}=E\left(4_{1}^{+}\right) / E\left(2_{1}^{+}\right)$ratio represents the rigidity limits of quadrupole collectivity being either rotational $(\sim 3.33)$ or vibrational $(\sim 2.0)$. Figure 4 shows the evolution of the low-spin states in the Dy isotopic chain. Inspection of the $2_{1}^{+}$and $4_{1}^{+}$states indicates that the vibrational $\rightarrow$ rotational phase transition takes place around $A=88$, and the $R_{4 / 2}$ values approach the rigid-rotor limit towards the middle of the neutron shell.

Low-lying non-yrast states are sensitive probes for microscopic structure of deformed nuclei. In general, the second $2^{+}$states $\left(2_{2}^{+}\right)$are associated predominantly with the bandhead of the $\gamma$-vibrational band. A qualitative explanation for the occurrence of the $\gamma$-vibrational $K^{\pi}=2^{+}$ state at relatively low excitation energy can be given in terms of the single-particle orbits in the deformed potentials; the operator $r_{2} Y_{22}$ acts on the single-particle states occupying a specific Nilsson orbit $\Omega\left[N n_{z} \Lambda\right]$ which satisfies the selection rule, $\Delta N=\Delta n_{z}=0$ and $\Delta \Lambda=\Delta \Omega= \pm 2$, giving rise to the $\gamma$-vibrational motions in the collective dynamics. In the region of interest, there are several pairs of orbits that meet the $r_{2} Y_{22}$ selection rule near the proton and neutron Fermi surfaces. The wave function of the $2_{2}^{+}$ state consists of a number of such two-quasiparticle excitations, which coherently contribute to the $\gamma$-vibrational amplitudes. Meanwhile, the interpretation of the excited $0^{+}$states $\left(0_{2}^{+}\right)$is rather complicated because there are several origins of the $K^{\pi}=0^{+}$excitations like the $\beta$ vibration, pairing excitations, two- $\gamma$-phonon vibration, and intruder states. The $K^{\pi}=2^{-}$states observed in the even Dy isotopes are interpreted as the band head of the octupole vibrational band with the predominant component $\pi^{2} 7 / 2^{-}[523] \otimes 3 / 2^{+}[411]$. 


\section{$K$-forbidden decays from multi-quasiparticle states ( $K$} isomers)

The region of well-deformed nuclei around $A=170-$ $180(Z \sim 70, N \sim 110)$ is known for the existence of lots of $K$ isomers [11,12], including those with half-lives longer than one hour. $K$ quantum number is defined as the projection of the total nuclear spin on the symmetry axis of the deformed nucleus. In addition to the spin selection rule, electromagnetic transitions connecting the states with different $K$ values $(\Delta K)$ are required to fulfill a selection rule that the multipole order of the transition $\lambda$ is equal to or greater than $\Delta K$, namely, $\lambda \geq \Delta K$. However, this $K$-conservation law is often violated due to various sources of symmetry breaking, such as Coriolis effects and softness in terms of the $\gamma$ (axially-asymmetric) degree of freedom, and hence strongly hindered transitions can take place. Such $K$-forbidden transitions are evaluated practically in terms of a reduced hindrance $f_{v}=F^{1 / v}$, where $F=T_{1 / 2}(\gamma) / T_{1 / 2}^{W}$ represents a hindrance relative to the Weisskopf single-particle estimate of the decay half-life, with the degree of $K$-forbiddenness defined by $v=\Delta K-\lambda$. It is known empirically that the value of $f_{v}$ ranges from 30 to 200 when $K$ is well sustained for both the initial and final states.

High- $\Omega$ orbits near the Fermi surface can contribute to the emergence of high- $K$ isomers at relatively low excitation energy. In general, a large (and robust) axial deformation yields good conditions for the formation of high- $K$ isomeric states. Thus, the discovery and characterization of such high- $K$ multi-quasiparticle states will shed light on various aspects of nuclear structure, such as intrinsic orbits near the Fermi surface, paring and other residual interactions, and the degree of axial symmetry in deformed nuclei. Furthermore, it is likely that high- $K$ isomers decays through low-lying excited levels not only in the groundstate band, but also in deformed-vibrational bands which represent crucial information for understanding the evolution of deformed structure.

This work focuses particularly on isomeric states that are expected to appear in ${ }^{170} \mathrm{Dy}(N=104)$ with $K^{\pi}=6^{+}$ and in ${ }^{172} \mathrm{Dy}(N=106)$ with $K^{\pi}=8^{-}$. The near-stability $N=104$ isotones are well known to have $K^{\pi}=6^{+}$isomers [13], which are interpreted as being based on the two-quasineutron configuration $v^{2} 7 / 2^{-}[514] \otimes 5 / 2^{-}[512]$. Configuration-constrained potential-energy-surface calculations for the $K^{\pi}=6^{+}$configuration predict the analogous isomer at about $1.2 \mathrm{MeV}$ in ${ }^{170} \mathrm{Dy}$ [14]. The large deformation and high degree of axial symmetry, predicted for both the two-quasineutron $K^{\pi}=6^{+}$state and groundband members to which the isomer decays, suggest that this nucleus may represent an extremely good example of a "pure" $K$ isomer [14]. Considering the $N=106$ isotones, $K^{\pi}=8^{-}$isomers which decay via an $E 1$ transition to the yrast $8^{+}$state have been identified over a broad range of proton number [13]. The $K^{\pi}=8^{-}$configuration is interpreted as the two-quasineutron configuration $v^{2} 7 / 2^{-}[514] \otimes 9 / 2^{+}[624]$. Based on the systematic behavior of the hindered $E 1$ decays [15], it is expected that the $E 1$ transition which de-excites the $K^{\pi}=8^{-}$isomer in
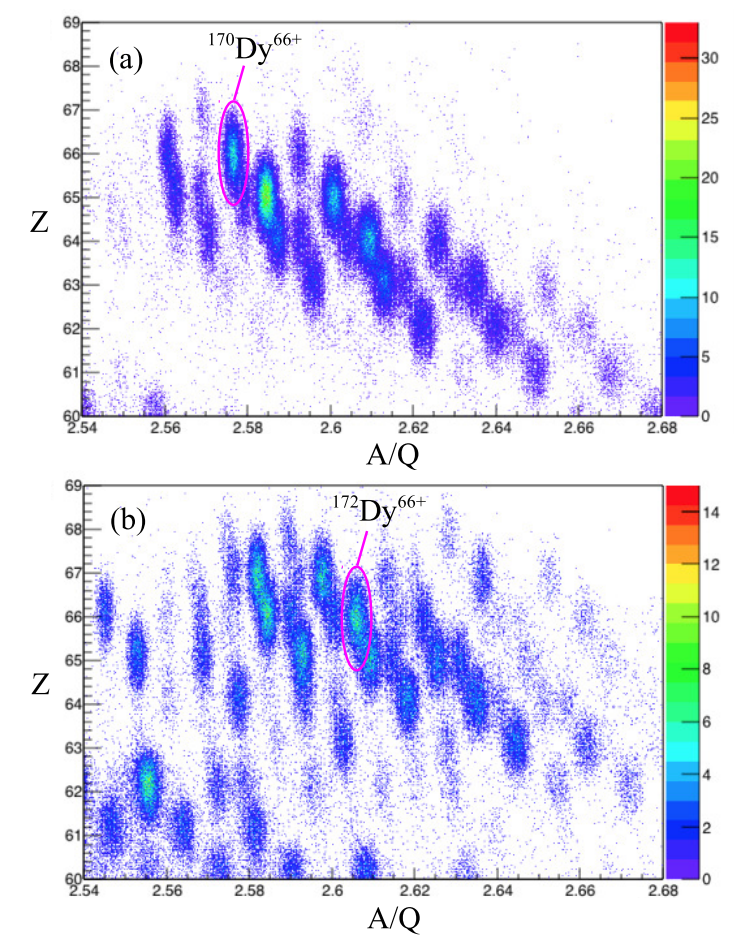

Figure 5. Particle identification spectra obtained with slit positions optimized for (a) ${ }^{170} \mathrm{Dy}^{66+}$ and (b) ${ }^{172} \mathrm{Dy}^{66+}$ ions.

${ }^{172}$ Dy will have a reduced hindrance of $f_{v} \approx 100$, resulting in a half-life of a few seconds. This is expected to be comparable to that of the ground state in this neutron-rich region, presumably leading to competition from $\beta$ decay in the isomer decay.

Neutron-rich rare-earth nuclei around $A=170$ were produced by in-flight fission of a ${ }^{238} \mathrm{U}^{86+}$ beam at 345 $\mathrm{MeV} / \mathrm{u}$ with the intensity of $12 \mathrm{pnA}$ on average, impinging on a beryllium target with a thickness of $5 \mathrm{~mm}$. The nuclei of interest were separated through the BigRIPS separator based on their physical and magnetic properties [16]. For purification of the secondary beams, wedge-shaped aluminum degraders with thicknesses of 4.5 and $1.0 \mathrm{~mm}$ were installed at the first (F1) and second (F5) dispersive focal planes, respectively. Identification of particles with the atomic number $(Z)$ and the mass-to-charge ratio $(A / Q)$ was achieved on the basis of the $\Delta E$-TOF- $B \rho$ method, in which the energy loss $(\Delta E)$, time of flight (TOF), and magnetic rigidity $(B \rho)$ were measured using the focal-plane detectors on the beam line. The secondary beams were transported with two different settings of the slits on the beam line; one is optimized for ${ }^{170} \mathrm{Dy}^{66+}$, and the other for ${ }^{172} \mathrm{Dy}^{66+}$. The obtained particle-identification spectra are shown in Fig. 5. About $8.3 \times 10^{3}{ }^{172}$ Dy ions were identified through the BigRIPS spectrometer.

The identified particles were implanted into a highly segmented active stopper system, named WAS3ABi [5], which consisted of two double-sided silicon-strip detectors (DSSSD) stacked compactly. Each DSSSD had a thickness of $1 \mathrm{~mm}$ with an active area segmented into sixty and forty strips (1-mm pitch) on each side in the horizontal and vertical dimensions, respectively. The DSSSDs also 


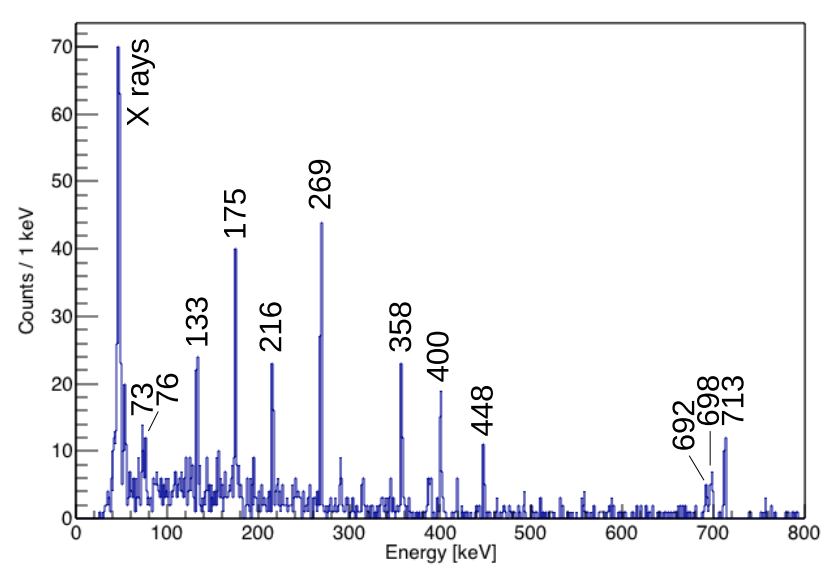

Figure 6. Gamma-ray spectrum measured in coincidence with electrons within $4 \mathrm{~s}$ after implantation of fully-stripped ${ }^{172} \mathrm{Dy}$ ions.

served as detectors for electrons following $\beta$-decay and internal conversion (IC) processes. Gamma rays were detected by the EURICA spectrometer [5], which consisted of twelve Cluster-type detectors, each of which contained seven HPGe crystals packed closely, which were developed for the former EUROBALL array. The $\gamma$-ray measurements were carried out with a time condition up to $100 \mu$ s relative to the trigger signal generated either from a plastic scintillation counter placed at the end of the beam line or from WAS3ABi.

The beam, electron, and $\gamma$-ray events were timestamped and recorded by independent data-acquisition systems. For the analysis of beam- $\gamma$ delayed coincidence, the $\gamma$-ray data sets were combined with those of the beam particles on an event-by-event basis using information on the time stamp. Isomeric states with (sub)microsecond lifetimes were identified with appropriate time gates. Meanwhile, all data sets containing beam, electron, and $\gamma$-ray events were used for $\beta-\gamma$ and IC- $\gamma$ coincidence analyses, in which the implantation of an identified particle was associated with the subsequent electron events that were detected in a given correlation area in the DSSSDs where the beam particle was implanted. Decay half-lives $\left(T_{1 / 2}\right)$ in the millisecond range were extracted from the time distributions of $\gamma$-ray gated electron events with respect to the fragment implantation.
Figure 6 exhibits a $\gamma$-ray spectrum measured in coincidence with electrons within 4 seconds after implantation of fully-stripped ${ }^{172}$ Dy ions. It is expected that the observed $\gamma$-ray spectrum consists of the transitions not only following the $\beta$ decay from the ground state, but also deexciting the long-lived $K^{\pi}=8^{-}$isomer that is predicted for the $N=106$ isotone as mentioned above. It will be possible to distinguish the internal and external decays by gating on time and energy spectra of electrons detected in the DSSSDs. The detailed analysis is still ongoing.

\section{References}

[1] Y. Yano, Nucl. Instrum. Methods B 261, 1009 (2007).

[2] T. Sumikama, K. Yoshinaga, H. Watanabe et al., Phys. Rev. Lett. 106, 202501 (2011).

[3] H. Watanabe, T. Sumikama, S. Nishimura et al., Phys. Lett. B 696, 186 (2011).

[4] H. Watanabe, K. Yamaguchi, A. Odahara et al., Phys. Lett. B 704, 270 (2011).

[5] S. Nishimura, Prog. Theor. Exp. Phys. p. $03 \mathrm{C006}$ (2012).

[6] A. Jungclaus, L. Cáceres, M. Górska et al., Phys. Rev. Lett. 99, 132501 (2007).

[7] L. Cáceres, M. Górska, A. Jungclaus et al., Phys. Rev. C 79, 011301 (2009).

[8] T. Otsuka, T. Suzuki, M. Honma et al., Phys. Rev. Lett. 104, 012501 (2010).

[9] R. Surman, J. Engel, J. R. Bennett et al., Phys. Rev. Lett. 79, 1809 (1997).

[10] S. Raman, C. Nestor and P. Tikkanen, At. Data Nucl. Data Tables 78, 1 (2001).

[11] P. Walker and G. Dracoulis, Nature 399, 35 (1999).

[12] F. G. Kondev, G. D. Dracoulis and T. Kibedi, At. Data Nucl. Data Tables 103-104, 50 (2015).

[13] G. Dracoulis, G. Lane, F. Kondev et al., Phys. Lett. B 635, 200 (2006).

[14] P. H. Regan, F. R. Xu, P. M. Walker et al., Phys. Rev. C 65, 037302 (2002).

[15] G. D. Dracoulis, G. J. Lane, F. G. Kondev et al., Phys. Rev. C 79, 061303 (2009).

[16] T. Kubo, Nucl. Instrum. Methods B 204, 97 (2003). 